\title{
Using Qualitative Research Software for CS Education Research
}

\author{
M. Dee Medley \\ Department of Mathematics and Computer Science \\ Augusta State University \\ Augusta, GA 30909 \\ USA \\ dmedley@aug.edu
}

\begin{abstract}
Research in Computer Science education has become more and more important in recent years. Both quantitative and qualitative research methods yield interesting results, but most researchers in our field rely on software for only the quantitative methods. This paper describes one of several packages on the market that support qualitative research methods. These packages make qualitative research less unwieldy and provide the researcher with excellent tools for doing far more detailed analysis of the data than is possible by hand. The data for such analysis may come from a variety of sources including on-line or written tests, programming assignments, and exit interviews for assessment purposes. The results of qualitative research can produce a better understanding of the larger picture in the environment under study.
\end{abstract}

\section{Introduction}

Research methods that are used for education include both qualitative and quantitative methods. In the past the latter have been far more widely recognized as providing support for theories of learning and pedagogy. However, the education community as a whole has been giving greater recognition to qualitative methods recently. The purposes for qualitative research projects are not the same as those for quantitative projects, but the information that these projects can yield may be no less important to us as professors. Qualitative studies tend to produce a better understanding of the larger picture in the target context.

\footnotetext{
Permission to make digital or hard copies of all or part of this work for personal or classroom use is granted without fee provided that copies are not made or distributed for profit or commercial advantage and that copies bear this notice and the full citation on the first page.

To copy otherwise, to republish, to post on servers or to

redistribute to lists, requires prior specific permission and/or a fee.

ITICSE 2001 6/01 Canterbury, UK

( 2001 ACM ISBN $1-58113-330-8 / 01 / 06 \ldots \$ 5.00$
}

Research projects that focus on a fairly large student population and have clearly defined questions to answer should use quantitative methods. Research projects that include small groups of students, seeks information on larger context, or tries to articulate questions for quantitative research may fit the qualitative paradigm. Much research in computer science education falls into the second category.

Qualitative techniques for analysis can be used for many projects that study Computer Science education. For example, a professor may want to know whether a new set of lab assignments provides a better method of teaching recursive techniques. Instead of simply counting the number of students who got a particular question correct on an exam or who handed in a completed programming assignment and using the results to generate some statistics, qualitative methods provide a way to look for patterns in students' misunderstandings. Qualitative methods are useful for analyzing students' answers to find trends and tendencies. For example, it may be useful to know that one's students misunderstand initialization methods in recursive functions, and this may not be easily seen by examining raw test or homework scores.

In addition, it may be fairly easy to collect data for qualitative studies. With many more on-line testing and grading methods, qualitative data can often be collected directly from tests. With programs, there is usually an electronic copy of their solutions that can be mined for data for a qualitative study.

It is also possible to use observational methods and online or face-to-face interviews for qualitative research. These methods of data collection can be used to produce textual data that can also be transcribed into text files for analysis. When the object of the research is to discover what students think or believe about their educational experiences in Computer Science, analysis their description of their experiences. Exit interviews to assess programs are exactly the sort of data for which qualitative methods are intended. 


\section{Purposes and Methods}

The goals, methods, sample selection, data collection, and findings in qualitative research tend to be different than similar characteristics of quantitative research. When the questions for the research are very clearly defined and can be measured using some specific criteria, quantitative methods are likely to be the best choice. Qualitative research starts with general questions and works toward an understanding of a situation.

Merriam [2] lists a number of attributes of qualitative studies that distinguish them from quantitative designs.

- Quantitative research starts from a definite hypothesis that must be proved or disproved. Qualitative research attempts to discover hypotheses, sometimes for the purpose of generating qualitative projects.

- Quantitative research yields very small, specific facts about clearly defined situations. Qualitative research tries to look at an entire environment or context and to find patterns within it.

- Quantitative research has specific methods for choosing samples to generate data. Qualitative research chooses samples from individuals that are the most likely to contribute to an understanding of the problem.

- Quantitative research uses instruments, tests, and objective means as data. Qualitative research uses interviews, essays, and observation as its data.

Strauss's [4] constant comparative style of analysis provides clear frameworks for analyzing this kind of data. As data collection proceeds, the researcher continuously reads, analyses and compares the data in an effort to discover patterns in the data. As this process continues, the patterns are given names called codes. As new data arrives, the researcher continues to examine it to assign these codes. Previously coded data must be reexamined and compared to the new data to discover new patterns or repetitions of old patterns. This process continues until a point is reached at which no new patterns appear.

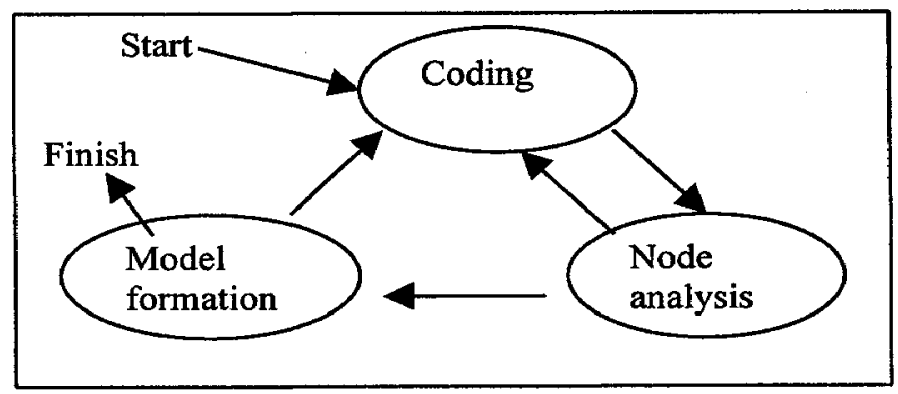

Figure 1. The Analysis Process
A good qualitative analysis software package allows the researcher to analyze a number of documents, to change and rearrange codes as patterns emerge, combine, and change, to find where the patterns occur in the data documents, and to form hypotheses in the form of models describing the findings.

\section{The NVivo Software Package}

Qualitative Software Solutions in Australia has two packages designed for doing qualitative analysis using the constant comparative method. The NVivo $[1,3]$ package is Windows-based, making it fairly simple to use immediately. It contains many more features than can be detailed here. A very minimal data set provides an example. The software allows analysis of data that is stored in the form of rich text files. This analysis produces a set of codes, which are patterns discovered in the data. The software collects the text that has been coded under each of the patterns and places each piece of text into a node that describes the pattern. The software can be used to develop models in the form of graphs that illustrate relationships among the nodes.

\subsection{A Small Example}

In our small example, answers to a question on an imaginary hour-long CS2 test covering recursion in linked lists form the data. Each imaginary student's answer has been placed in a file and saved in rich text format. The intention of the imaginary researcher is to discover which concepts of recursion in linked lists the students in this class misunderstood. The coding discovers common errors and links answers that display similar problems in understanding.

\subsection{Coding}

Coding the data consumes the major portion of the analysis time. Once the documents containing data have been coded other features of NVivo make it simple to organize and understand the emerging results. This example includes a single file of answers to a test question, but a real situation might require more files to obtain useful results.

Each file in a project must be in rich text format. The software allows changes to the files during coding, so it is not only possible to code existing files, but also to include memos and commentary as the analysis proceeds. The researcher reads the text and considers what the text tells about the situation. Highlighting text in the file makes it possible to attach a code to that portion of the file. For example, misuse of a value parameter might be coded as a specific error. This error might lead to a final analysis that students fail to use parameters correctly in their recursive programs. 


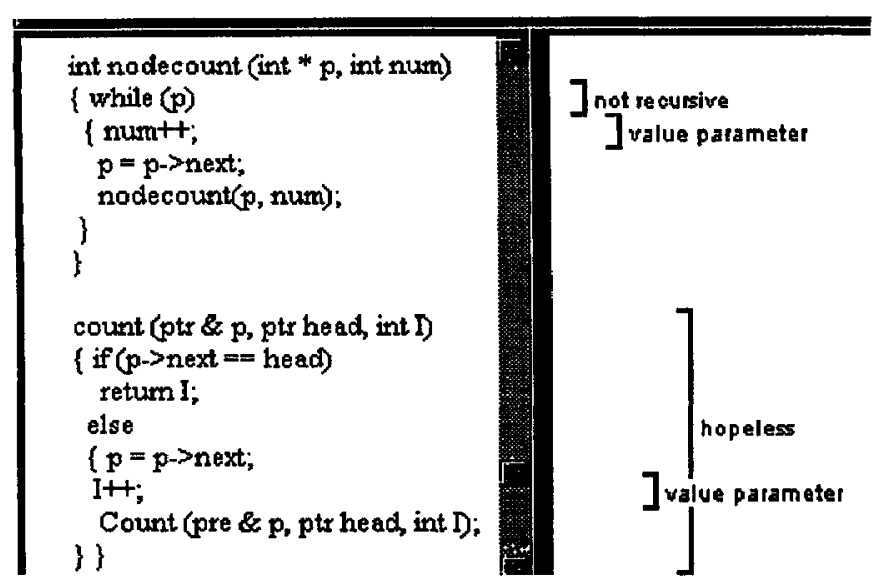

Figure 2. Coding a text file.

Patterns emerge as the researcher codes passages in the text by highlighting passages and selecting a code. The file can be viewed with the codes indicated in the right margin (Figure 2.) In our example, some of the codes that have been found are:

- not recursive

- not initialized

- value parameter

- hopeless

Each of these codes is represented by a node in the next stage of the analysis. These codes tend to change and rearrange as the analysis phase proceeds. Codes may initially appear to describe the patterns found in the data but may later split into two or more different codes. And some codes that initially appear to describe patterns may not seem useful at a later stage of the analysis. The software makes it fairly simple to add codes and to completely recode the text files.

\subsection{Working with Nodes}

The software makes it easy to find the coded parts of the text once a set of codes has been created. Since there may be many different files of data involved in a larger study, the codes may be spread around each of them. The software finds and collects the pieces of data that match each of the codes.

The software provides a facility for working with the codes as individual collections of data. Figure 3 illustrates an example of one such collection. In our imaginary test, some students made the mistake of using a while statement in their supposedly recursive functions. This mistake is coded "not recursive."

It may not have been clear initially that "not recursive" was a single pattern or that another wording might have been more descriptive. But the process of coding and then examining the collection of text associated with that code helps to clarify the descriptiveness of the code.
When the analysis produces a fairly complete set of codes these codes are arranged into nodes to form a collection of snippets of text that can be used for describing the findings of the research. Each node includes the names of the files that contain data that was coded as part of that node, the section and paragraph where the coding occurred, and the number of characters that were coded with that name. The software thus provides an extremely simple method for quoting the data in order to write up the findings of the study.

Document 'qual res', 3 passages, 41 characters. Section 0, Paragraph 144, 14 characters.

while $(1 !=1)$

Section 0, Paragraph 154, 17 characters.

while $\mathbf{p} !=$ head)

Section 0, Paragraph 171, 10 characters.

while (p)

Figure 3. A Node Example (Not Recursive)

\subsection{Creating Models}

Once the text has been thoroughly analyzed and the patterns have been established as nodes, the researcher may want to find relationships among the patterns. The software provides a way to do this using graphs. The nodes form the vertices of the graphs, and the edges illustrate the relationships among the nodes. These relationships may be undirected or directed. These models can be used for further analysis of the data to determine whether there is really a good fit.

In our simple example, a relationship was discovered between answers that were hopeless, or that showed little evidence of understanding recursion with linked lists, and answers that were not recursive, or that attempted to use while loops. Another relationship was discovered among three codes or patterns. Students who failed to pass a counter by reference (by value instead) also failed to understand how to return results and how to initialize accumulators in their recursive functions. These relationships are modeled in Figure 4. 


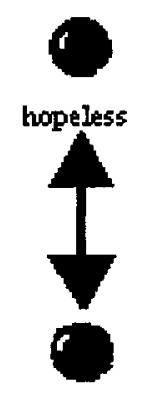

riot recursire

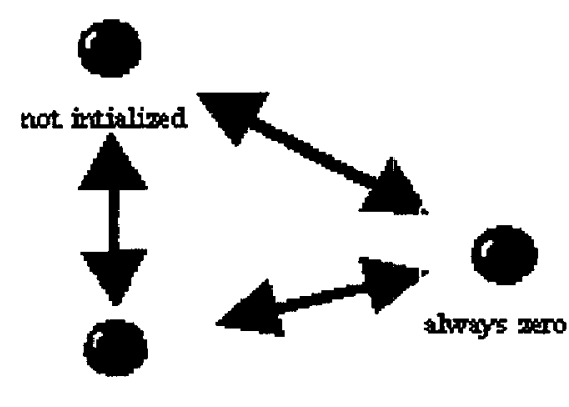

vrable parameter
Figure 4. A Simple Model

The software allows the user to try different ways of organizing the nodes to show how the various patterns fit into a whole picture. Many different models can be constructed this way until one is found that describes the findings clearly. The software allows color coding, multiple styles of arrows, and other methods to create clear visual models of complex structures and relationships.

During the research process it is always possible to return to the coding stage to find more or different patterns. After the coding appears to be complete the researcher will look at the nodes individually to see if the data coded into that node really describes a pattern. It may be that some of the data actually fits a different pattern. Or it may be that the current code does not completely describe the pattern found. It is also possible that the data that has been coded into a node actually forms two patterns, and the researcher then must return to the coding stage to recode using the new patterns.

Using the nodes to form models can assist understanding what the patterns are as they emerge during the analysis. When the researcher attempts to find relationships among the nodes, new patterns may emerge that may require returning to the coding stage to refine the number or names of the nodes.

\section{Discussion}

This kind of software provides a real service for anyone who is attempting to do discovery research. It has always been possible to find good packages for quantitative research, but AI methods and the GUI interface have made qualitative research methods equally viable. The NVivo software not only makes this form of research easier to do, but using it helps to foster new ideas about how the patterns change, flow, and relate to each other.

Research in Computer Science education has been lacking in the past but recently there has been a surge of interest in better research techniques in the pedagogy of our discipline. Qualitative research methodology does not perform well in the same circumstances or the same way that quantitative methods do. However, quantitative methods are not workable for hypothesis formation since their purpose is to support hypotheses. Qualitative designs provide methods for hypothesis creation. All research projects must begin with the formation of a hypothesis. Too often such hypotheses are formed by guesswork or by a desire on the part of the researcher to support a particular pedagogical method or tool. The use of qualitative methods for hypothesis formation makes the front end of the research process far more controlled.

Another major purpose for qualitative research methods is the study of small groups. It is not usually possible to generalize the findings of a qualitative study. But when studying a small group generalization of findings is not the purpose. Quantitative methods do not provide many resources for such studies. However, qualitative methods are quite useful for this kind of research project.

Another example of a qualitative style of study occurs in assessment procedures for departments. Many departments conduct exit interviews of students for their own use. The data collected in these interviews is often summarized briefly and then forgotten. If the interviews are taped, they can be transcribed and analyzed using software such as NVivo. Additional years of data can be added as it is acquired to determine whether needed change in departmental methods or curriculum has taken place. There is no need to generalize the findings of this kind of study, but the results are important to the continued success of a department.

\section{Conclusions}

Qualitative methods are not appropriate for all kinds of educational research, but they clearly have a number of applications to some of the projects that are useful to Computer Science. The use of software to support the analysis in these studies can enhance the reliability of the findings simply by making it easier to work with the data until good results are available. The NVivo software provides a good tool for analyzing qualitative data.

\section{References}

[1] Fraser, D.: NVivo Reference Guide, Qualitative Solutions and Research, 1999.

[2] Merriam, S.: Qualitative Research and Case Study Applications, Jossey-Bass Publishers, 1998.

[3] Richards, L.: Using NVivo in Qualitative Research, Sage Publications, 1999.

[4] Strauss, A. L.: Qualitative Research for Social Scientists, Cambridge University Press, 1987. 